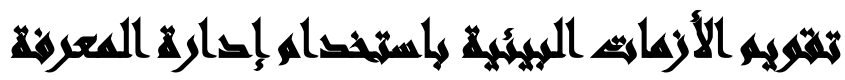

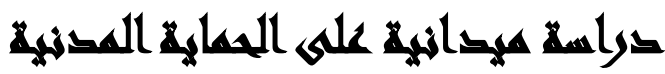

\section{[IY]}

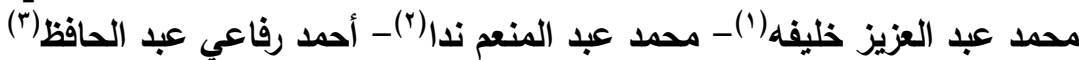

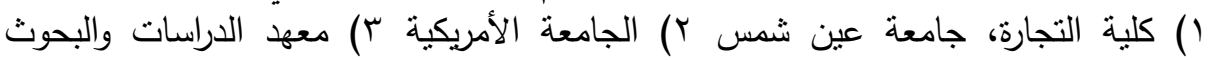

البيئية، جامعة عين شمس جامعة

\section{المستخليف}

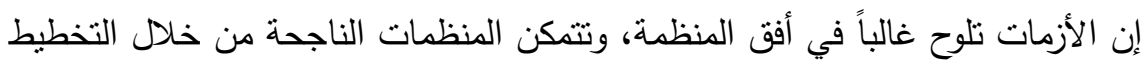

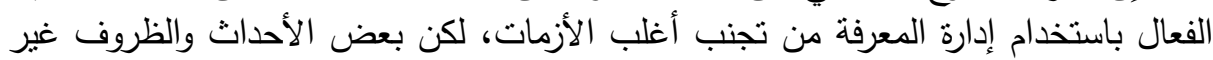

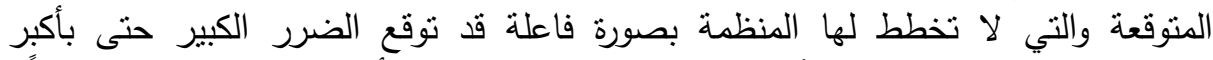

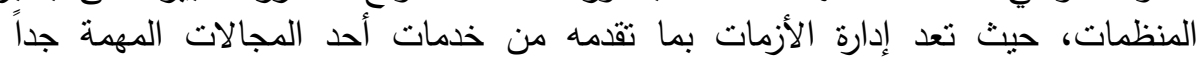

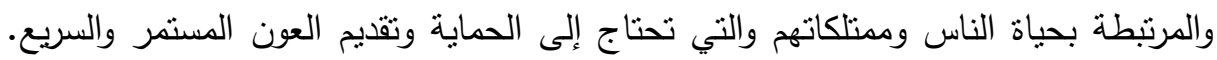

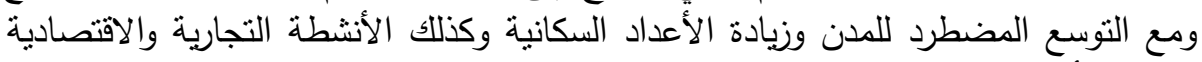

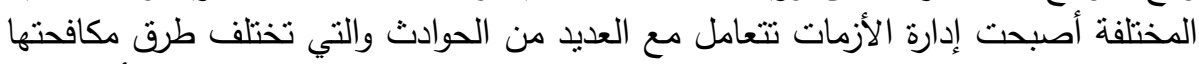

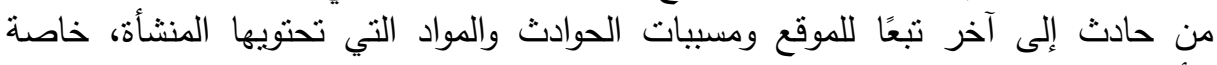

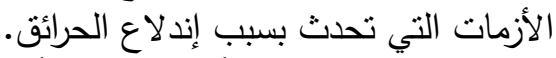

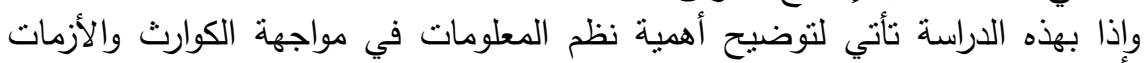

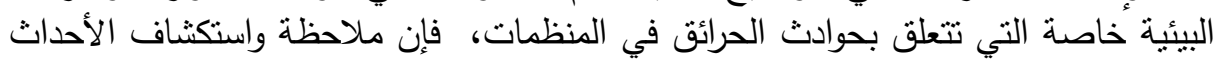

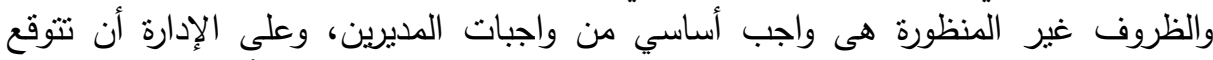

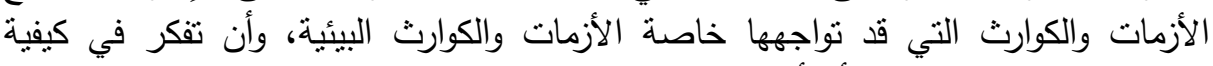

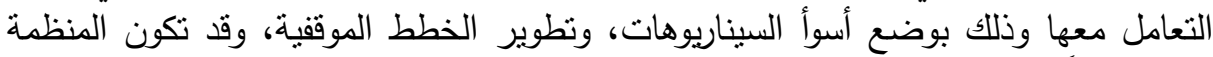

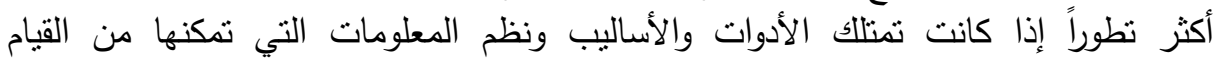

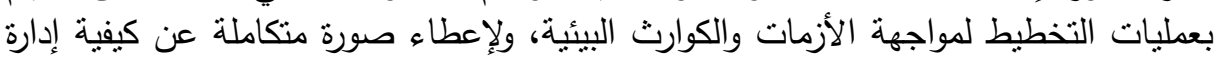

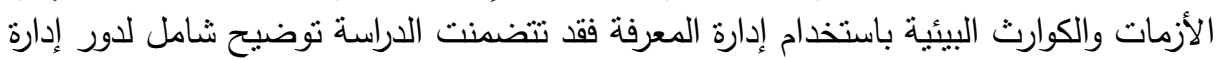

حيث شملت الدراسة لدفهوم وأهمية إدارة المعرفة ودورها في الارتقاء بمسنوى الأداء،

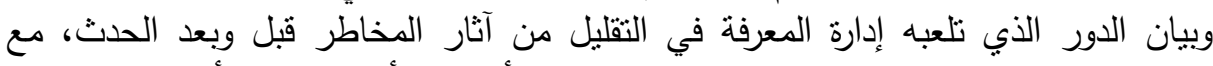

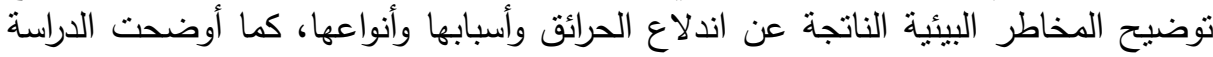

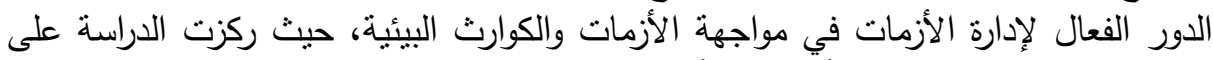

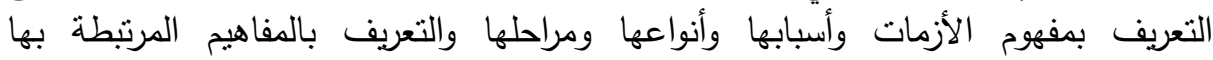

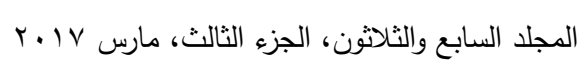


والمنداخلة معها، كما ركزت الدراسة على تحديد ماهية إدارة الأزمات، وكذلك بيان دور نظم

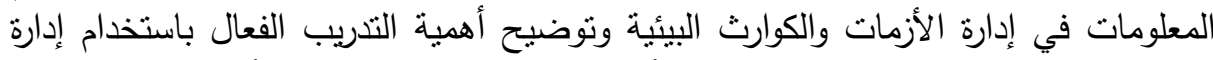
المعرفة لتقعيل الدور الذي تقوم به إدارة الأزمات في مواجهة الكوارث والأزمات البهات البيئية داخل

وقد خلصت الدراسة إلى عدة نتائج أهمها الدور الفعال الذي تلعبه إدارة الأزمات البيئية

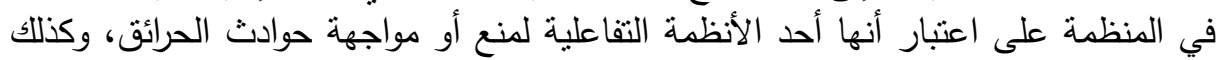

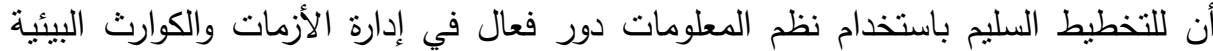
وإعداد سيناريو إدارة الأزمات والكوارث البيئية على أسس علمية سليمة.

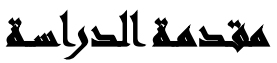

تثشير الدراسات الخاصة بعلم دراسة الكوارث تكرارية حدوث أنواع متعددة من الكوارث

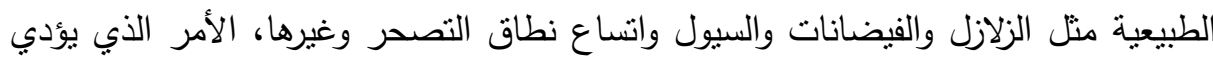

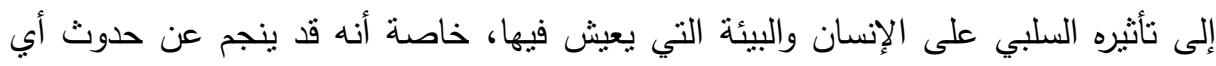
كارثة طبيعية تلوث بيئي بالمنطقة التي حدثت بها الكارثة، مما يهدد بحدوث مشكلات طويلة بلئل

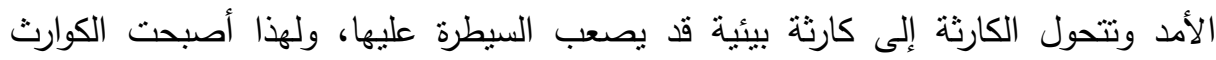

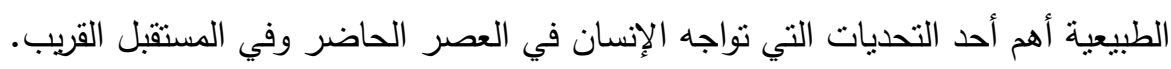

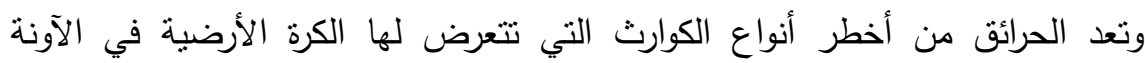

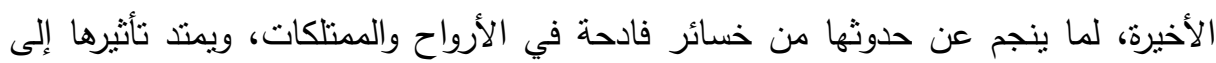

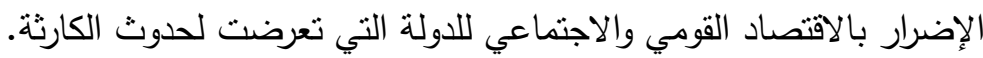
ومن هنا كان من الضروري الاهتمام بالأسلوب العلمي في دراسة الحرائق ومهارات

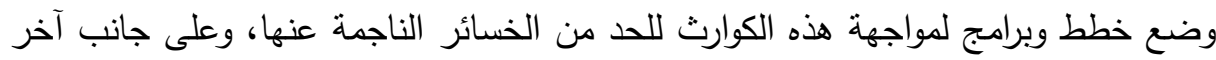
تدبير وتطوير الوسائل العديدة التي تخفف من الآثار الناتجة عن حدوث الكناثِ الكوارث الطبيعية، وتضافر جهود العلماء بدراسات الكوارث الطبيعية على المستوى الإقليمي والعالمي. وتعد إدارة الكوارث البيئية إدارة علمية صحيحة يمكن من خلالها الخروج من الكارثة بأقل

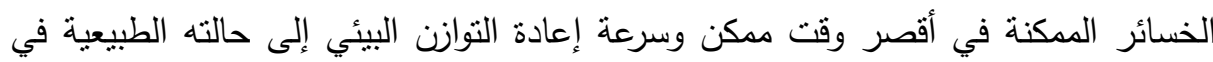
مسرح الكارثة، وتهدف هذه الدراسات إلى إلقاء الضوء على الحرائق وأبعادها وخلفياتها وأهدافها 
ومتطلبات إدارتها وبيان دور استخدام إدارة المعرفة ونظم المعلومات في تقويم مثل هذه

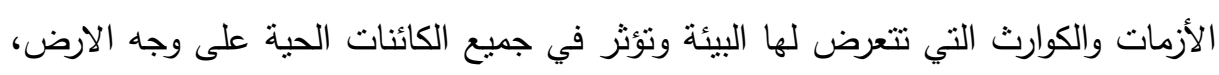

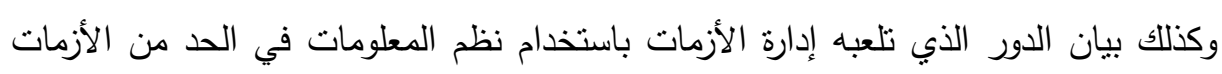

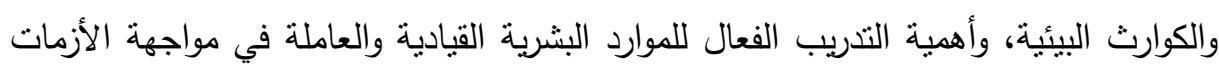
البيئية في أى منظمة.

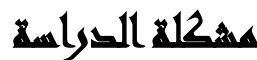

تركزت مشكلات البحث في انخفاض مستوى استعداد المنظمات الحكومية وغير

الحكومية في مواجهة الكوارث نتيجة عدم تطبيق منهجية الإدارة في التفاعل مع الأزمات، لها تقوم ثلك المنظمات برد فعل عشوائي في مواجهة الكوارث، الأمر الذي يؤدي إلى زيادة الآثار

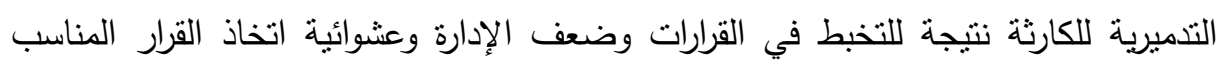

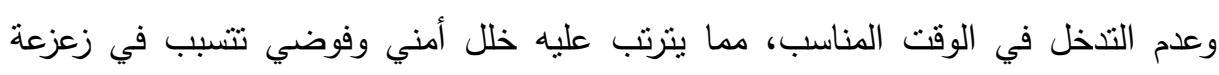

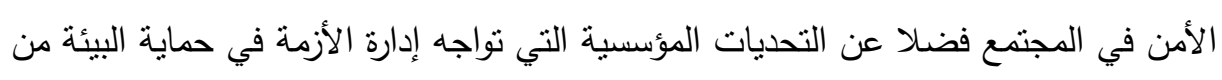

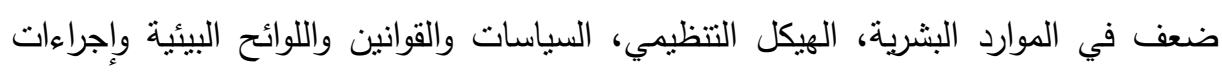
الإدارة البيئية، وكذا المسائل المالية، وذللك نتيجة لعدة أسباب أهمها: - عدم وجود خطط للاستعداد والمواجهة وإن وجد تكون غير محدثة أو غير معلنة.

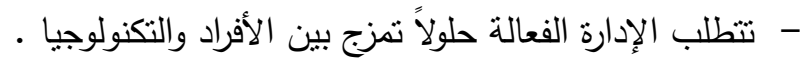

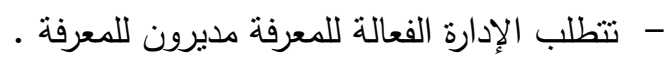
- فوائد إدارة المعرفة وضع خطط لها أكثر من وضع نماذج للمعرفة. - - إدارة المعرفة عملية مستمرة وليست لها نهاية.

وسوف يقوم الباحث بمعالجة كارثة الحرائق باستخدام إدارة المعرفة والتي تتضمن الآتي:

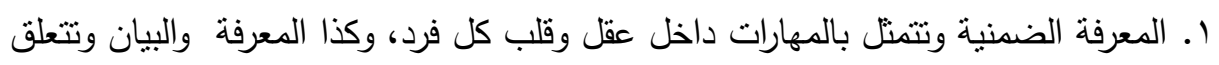

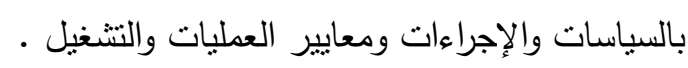
r. تضيف إدارة المعرفة مبادئ أساسية لها وهى تساهم في تقديم وحل الأزمات البيية. 
r. من خلال أنواع الأصول المعرفية (رأس المال الفكري)، والمتمنلة في أصول المعرفة (التجريبية المفاهيمية النظامية العامة) يمكن التوصل إلى حلول متوازنة تحد من من ظاهرة كارثة الحرائق.

ع. خلق المعرفة يتطلب نتاول عمليات الإدارة وهى العملية الاجتماعية والتجسيدية والتوافقية والذاتية والابتكارية.

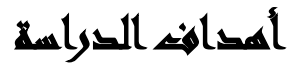

ا ـ استعراض الأزمات والكوارث البيئية الخاصة بالحرائق وأسبابها والتصرف فيها.

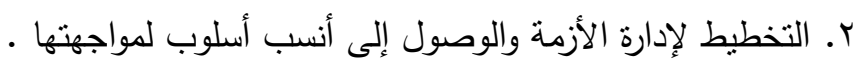

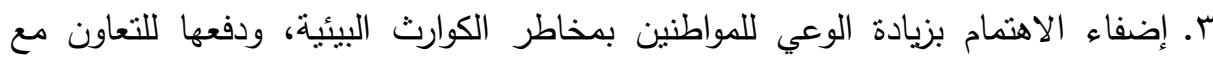
الجهات المعنية بإدارة الكارثة للحد من المخاطر المحتملة وحماية أنفسها وممتلكاتها من

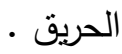

ع. وضع إطار مقترح لتقديم الخدمات البيئية الناتجة عن الحريق باستخدام إدارة المعرفة . ه. إجراء دراسة تطبيق موضوع البحث على إدارة الحماية المدنية.

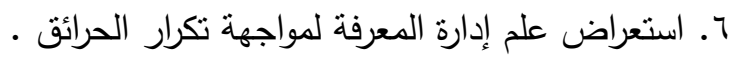
V. القيام بدراسة نطبيقية لاختبار فروض البحث والتوصل إلى النتائج والتوصيات الإدارية لتظوير النظم الإستراتيجية، بما يؤدي إلى زيادة تفاعلها قبل وبعد الأزمات البيئية . لئه

\section{أهمية التواسمة}

تكمن أهمية البحث في أن ظاهرة تكرار كوارث الحرائق يحتاج إلى أسلوب جديد لتطبيق

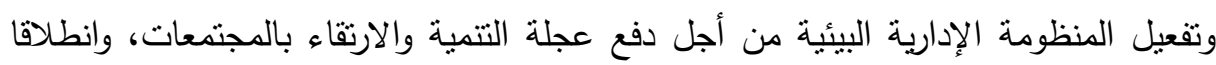
من مشكلة البحث نجد أنها تتطوي على أهمية نظرية وتطبيقية يمكن بلورتها كما يلي: 
بالنسبة للمجتمع: أن مواجهة الكوارث المختلفة ينطلب أساليب عصرية لكي يضمن

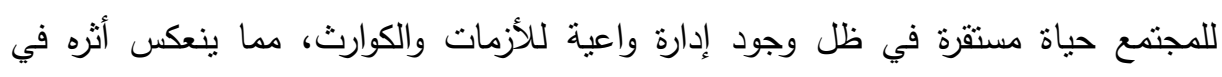

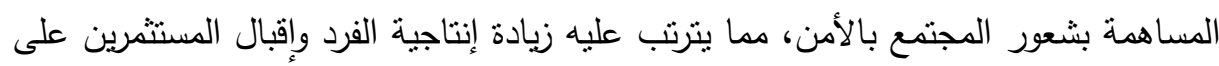

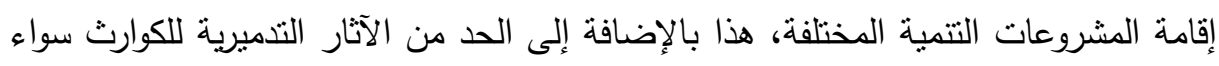

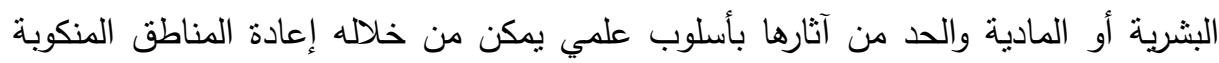
بسرعة دون انتثار الفوضى والمساعدة في دفع عجلة التتمية والاستقرار الأمني.

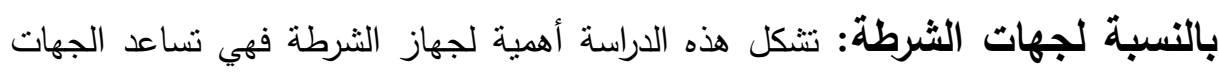

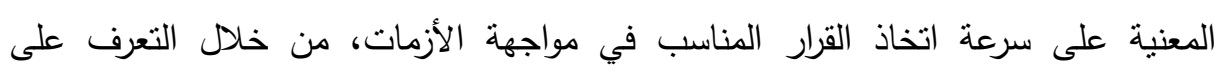

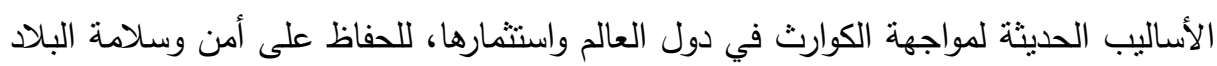

$$
\text { في الأزمات والكوارث. }
$$

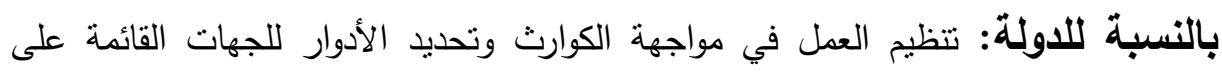

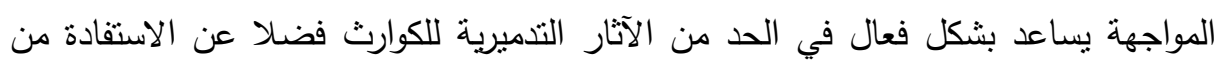

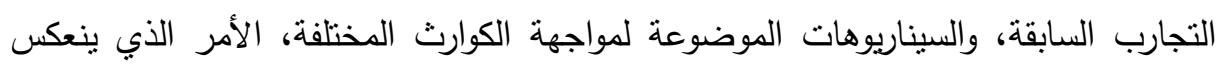

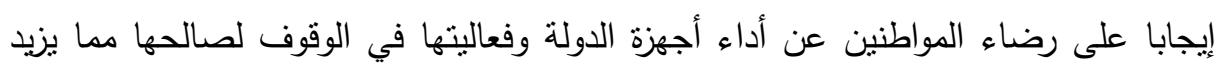

$$
\text { بشعورهم في الانتماء للدولة. }
$$

\section{هغوضر الفراسما}

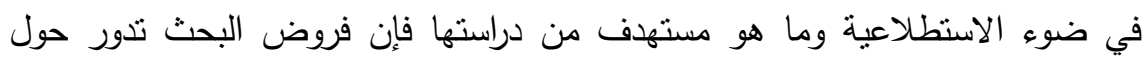

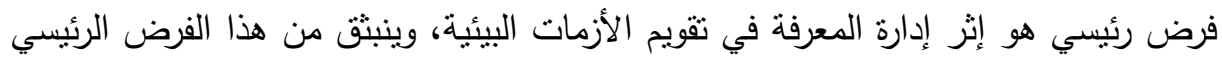

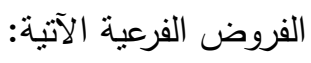
الفرض الأول: توجد علاقة ذات دلالة معنوية للأنظمة المطبقة حاليا لمنع الحرائق وبين ارتفاع معدلات الحريق. الفرض الثاني: توجد علاقة ذات دلالة معنوية بين الدنطلبات البيئية المطلوبة للحد من الحرائق وإمكانيات المنشآت المادية والبشرية. 
الفرض الثالث: نوجد علاقة ذات دلالة معنوية بين إدارة المعرفة وإدارة الأزمات البيئية الناتجة

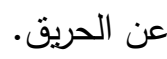

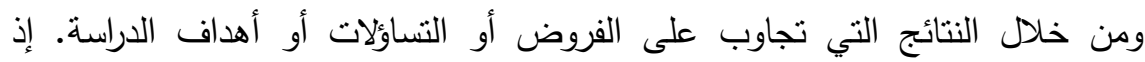

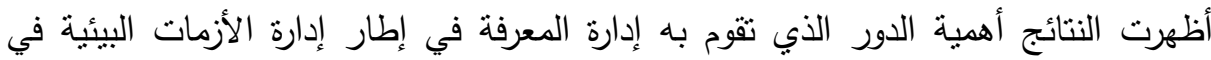

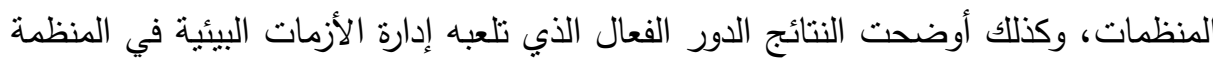

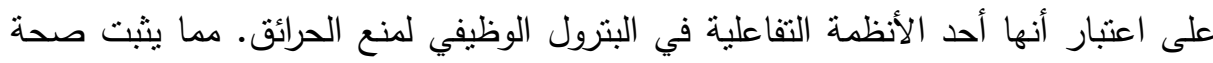

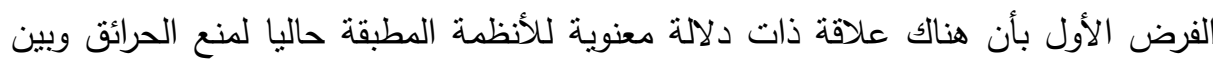

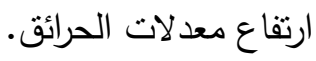

كما أثنارت النتائج إلى أهمية التخطيط ودوره في إدارة الأزمة البيئية باعتبارها من

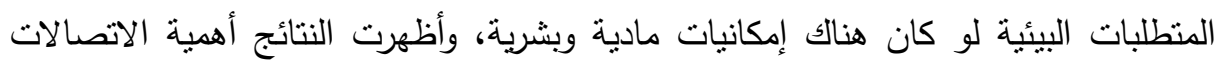

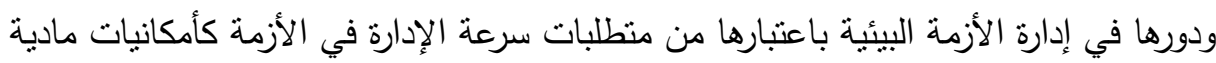

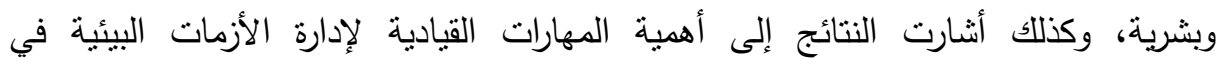

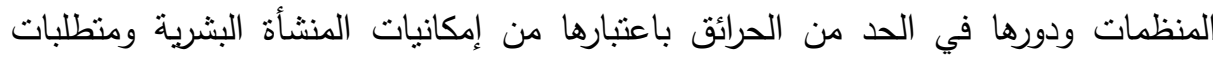

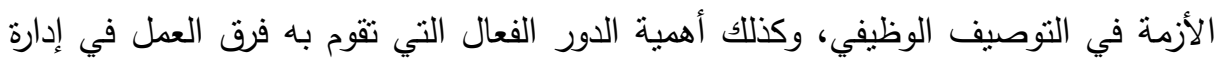
الأزمات البيئية. مما يثبت صحة الفرض الثاني بأن هناك علاقة ذات دلالة معنوية بين المتطلبات البيئية المطلوبة وإمكانيات المنشأة المادية والبشرية للحد من الحرائق.

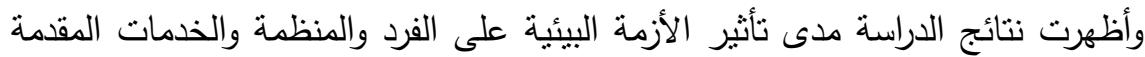
وضرورة معرفة معايير النظم البيئية المطبقة لمعرفة ددى الاحتياج لتطبيق إدارة المعرفة للتقليل من مخاطر الأزمات البيئية، كما أوضحت النتائج مدى اعتماد المنشأة في تنوزيع النئه

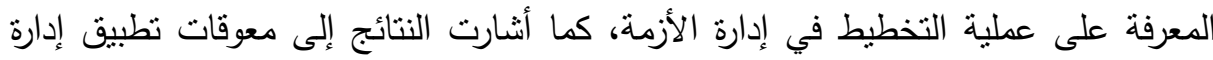
المعرفة لتقويم الأزمات البيئية في المنظمات والتي تشمل كفاءة قياس الإكانيات الإدهات المادية والبشرية وأجهزة الإنذار المبكر والتدريب والإدارة الفعالة والإدراك الفعلي لمعنى إدارة المعرفة،

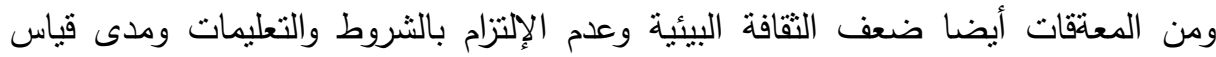
المعوقات الفنية والتدريبية والغدارية والتي تؤثر بشكل أكبر على مخاطر الحريق، وكذلك بلك

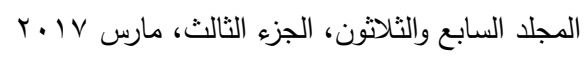


أوضحت النتائج مقومات إدارة الأزمة البيئية للتقليل من مخاطر الحريق في المنظمات والتي

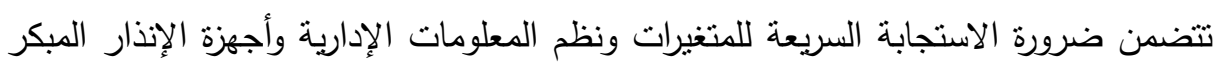

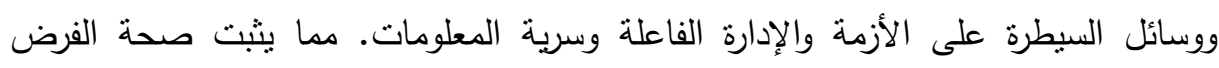
الثالث وهو وجود علاقة ذات دلالة معنولة بين إدارة المعرفة وإدارة الازمات البيئية الناتجة عن الإداعة الحريق.

\section{منهمج الترواسلة}

تتطلب البحوث العلمية نوعا من التفكير الذي يتضمن كلا من المنهجين الاستقرائي

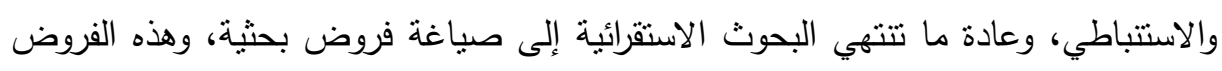

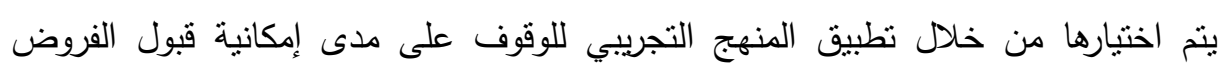
البحثية ويعتمد هذا البحث على منهجين أساسيين هما:

أ. المنهج الاستقرائي: يهدف إلى بناء إطار لأبعاد مشكلة البحث وأهدافه، حيث أنه النه

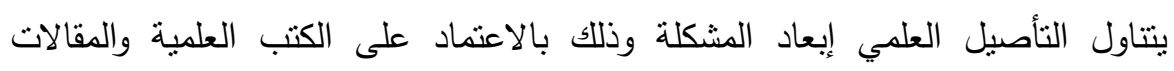

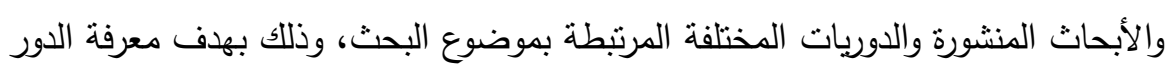
المتوقع القيام به من قبل، للحد من وقوع الكوارث البيئية والذي يتحكم فيها الإنسان وبخاصة الحرائق.

ب. المنهج الاستنباطي: يقوم الباحث من خلاله باختبار فروض البحث وتحديد مدى قبول هذه الفروض من عدمه، وتقييم الدور الذي يمكن أن يلعبه المسئولين عن اندلاع تلكي

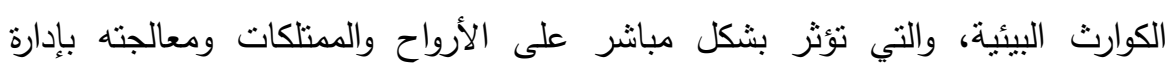

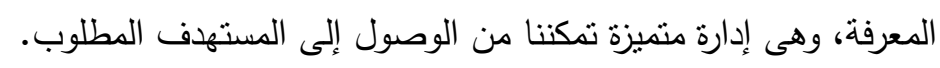

\section{مشوض الفراسم}

تم تركيز الدراسة على الحرائق باعتبارها أحد الكوارث التي ينتج عنها خسائر في الأرواح

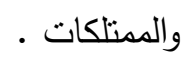




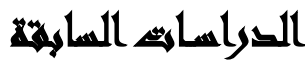

لقد وجد الباحث صعوبة في البحث في الحصول على دراسات لقلة الدراسات الوطنية في هذا المجال (على حد علم الباحث) لهذا السبب ومن أجل البدء مما انتهى إليه الآخرون فقد قام الباحث بمراجعة قواعد المعلومات واستشارة عدد من المهتمين في هذا المجال، وزار عدد من المكتبات، وتوصل من خلال هذا الجهد المبذول إلى الحصول على بعض الدراسات السابقة ذات العلاقة المباشرة والنسبية بموضوع الدراسة، وحيث أن تطبيق إدارة المعرفة في المنظمات لحل الأزمات البيئية يكون بنفس الخطوات مع وجود اختلافات بسيطة في نوعية الأزمات التي تتعرض لها المنظمة وكيفية إدارتها، باختلاف طبيعة عمل المنظمة، فجميع هذه المنظمات (تجارية وصناعية وتعليمية وصحية وأمنية ...) تعمل في بيئة أصبح التطور والتغير والتجديد سمتها الأساسية والاستجابة لهذا التطور ضرورة ملحة، فتعتبر إدارة المعرفة إحدى الوسائل الأساسية التي تمكن هذه المنظمات من الوصول إلى مرحلة التميز، وقدابه

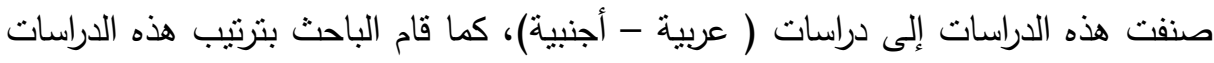
طبقا لزمن إصدارها من الأقدم إلى الأحدث. ففي دراسة سالم الصغير وهى بعنوان "تماذج من إدارة الأزمات في القرآن الكريم" فقد هدفت إلى توضيح المنهج القرآني لمعالجة الأنواع المختلفة من الأزمات، وإثبات إسهام الثقافة

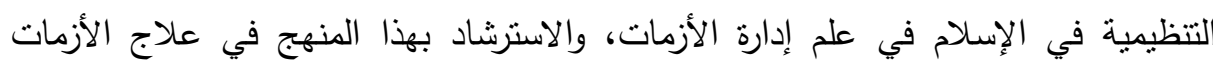
المشابهة في العصر الحديث. ونتيجة لأهمية هذه الدراسة في إضافة رؤية جديدة في علم إدارة الأزمات، فقد كانت المنهجية المستخدمة عبارة عن دراسة متعقة في القرآن وكتب التفسير والفقه والتاريخ، بالإضافة إلى كتب إدارة الأزمات والأبحاث العربية الأجنبية. وقد نوصلت فئه الدراسة إلى مجموعة من النتائج من أهمها أن القرآن الكريم قد بين من آلاف السنين كيفية

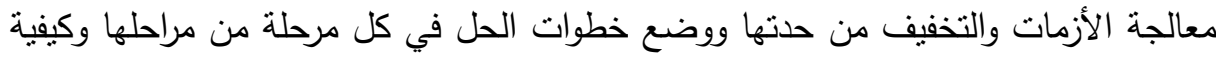

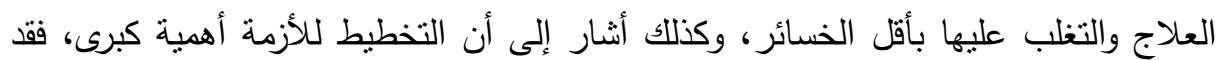

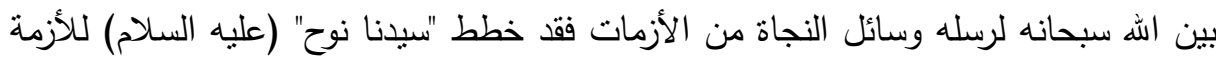
بصنع السفينة، وخطط "سيدنا يوسف" (عليه السلام) لأزمة مصر لمدة خمسة عشر سنة لهاه 
بالاتتاج والتخزين وترشيد الاستهلاك، كما بين القرآن الكريم أن للقيادة أهمية كبرى في إدارة

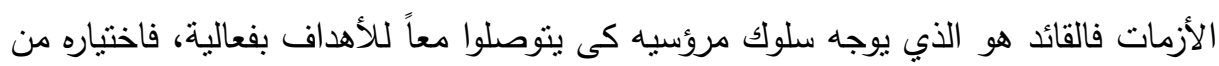

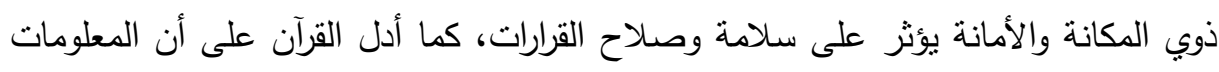

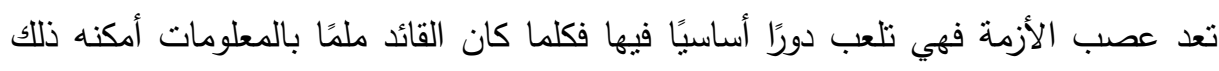

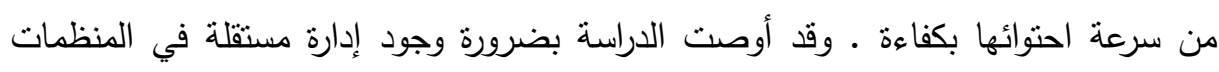
تكون مهوتها جمع المعلومات والتتبؤ ووضع الخطط والسياسات والبرامج والسيناريوهات لكأزمات المقبلة.

وتبين من دراسة (ميخائيل) وهى تحمل عنوان دور الوعي الاجتماعي في مواجهة الأزمات والكوارث البيئية- دراسة ميدانية لاور الوعي الاجتماعي في مواجهة أزمات التلوث البيئي في محافظة المنيا"، أن للوعي الاجتماعي دورًا هامًا في التقليل من آثار الأزمة البيئية

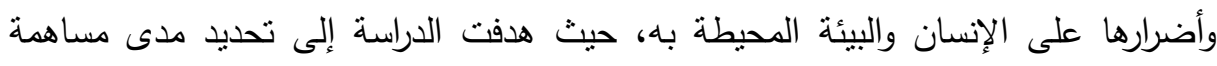
الناس في التصدي للتلوث البيئي وتحديد نوع هذه المساهمة والمعوقات الني تعوقهم عن ذللك. كما توصلت الدراسة إلى أن السبب الجوهري في حدوث أزمة التلوث البيئي في مجتمع الدراسة

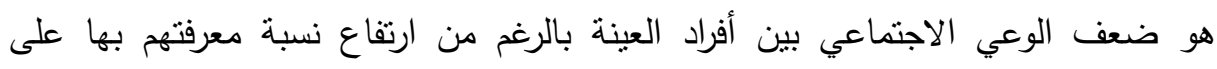

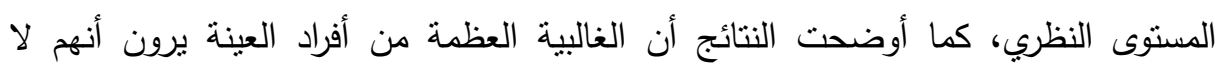

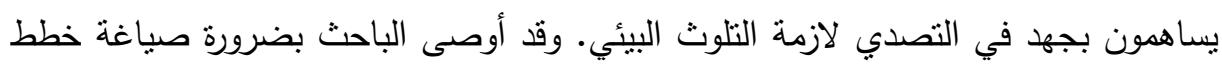
لتوعية الناس للتعامل السليم مع الأزمات والكوارث البيئية، وذللك من خلال برامج إعلامية لئه

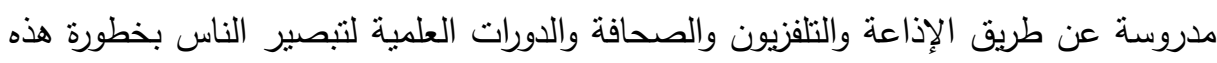
الأزمات والكوارث وكيفية استجابتهم لها وأساليب تصرفاتهم معها إيجابيًا،

وفي دراسة (فراج وحنفي) وهى تحمل عنوان السلوك الجماهيري في مواجهة الأزمات والكوارث - دراسة سوسيولوجية لمنطقة حريق الحي التجاري ببورسعيد"، حيث تستهدف هذه

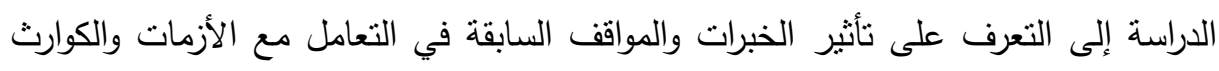

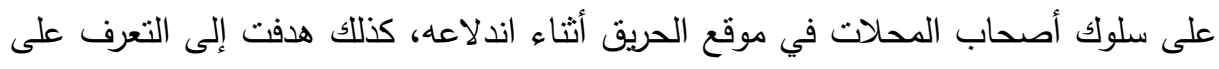

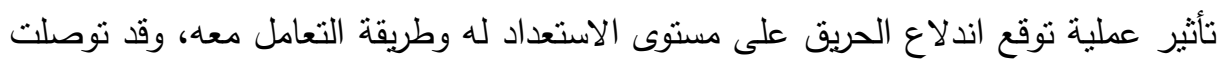

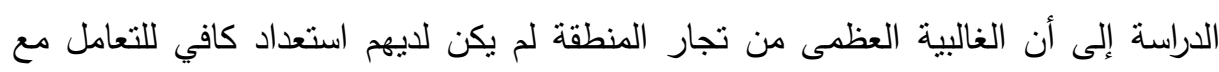

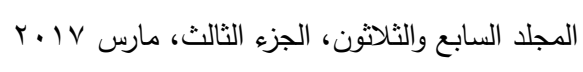


الحريق، حيث أنه لم يكن لديهم طفايات للحريق في محلاتهم، وقد أوصى الباحث بضرورة

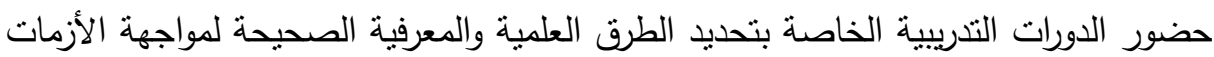
والكوارث التي تحدثها حوادث الحريق. وقد تبين من دراسة (الملكاوي والعمري) وهى بعنوان "دور إدارة المعرفة في التقليل من آثار المخاطر (دراسة نظرية)، أن إدارة المعرفة تلعب دوراً مهماً في التقليل من الآثار السلبية للخطر الحاصل من خلال توفير البيانات والمعلومات اللازمة التي تمكن المنظمة من التعامل مع المخاطر بالطريقة السليمة بحيث تصل آثارها السلبية إلى أدنى درجة، وفي أحيان كثيرة فان إدارة المعرفة تمكن المنظمة من تجنب الخطر بشكل كامل بفعل التنبؤ المسبق

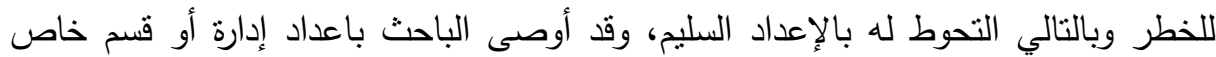
بالمنظمة (خاصة المنظمات التي تواجه مخاطر نتيجة لطبيعة اعمالها)، حيث يتولى ذللك القسم جمع البيانات والمعلومات ذات العلاقة وتحليلها والتتبؤ بطبيعة ونوعية الاحداث لتحديد نوعية الخطر المحدق، وكذلك توثيق جميع البيانات ذات العلاقة بالمخاطر وكيفية التعامل

Validating A unified framework for " وفي دراسة لـ (كارولين) وهى بعنوان Knowledge Management" عبارة عن دراسة مسحية أجريت مع عدد من الباحثين والمديرين في عدد من قطاعات الأعمال

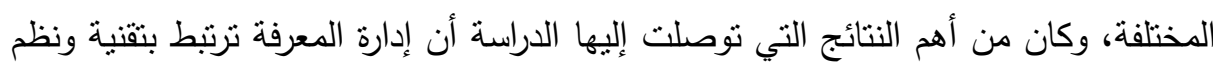

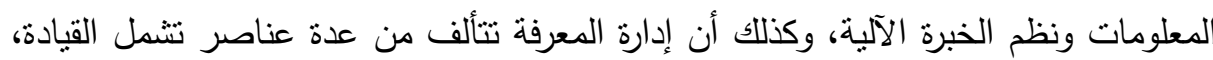

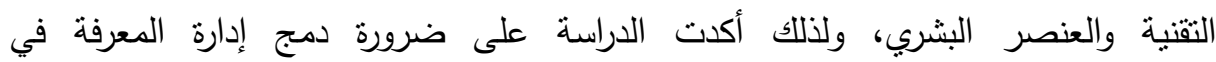
التخصصات الأكاديمية في الجامعات. Chaos, crises and disasters: A وهى بعنوان (Brent) وما تبين من دراسة :strategic approach to crisis management in the tourism industry" "الفوضى، الأزمات والكوارث: نظرة إستراتيجية لإدارة الأزمات في قطاع السياحة"، ضرورة تمتع المنظمات بالمرونة والرقابة المستمرة والتوجه نحو تصميم وتطبيق استراتيجيات فاعلة لإداته 304

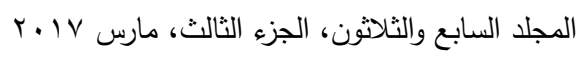


للتعامل مع الأزمات. كما أكدت على ضرورة استخدام المنظمات للمدخل الثمولي في إدارة

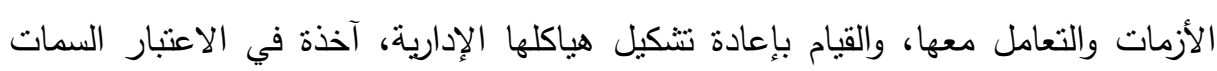
المرتبطة بتخصيص الموارد والثقافة التنظيمية .

ويرى الباحث أن الدراسات السابقة جاءت مؤكدة على أهمية إدارة المعرفة، وأهمية الدور

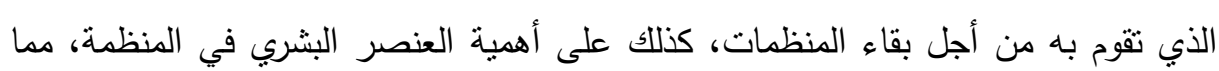

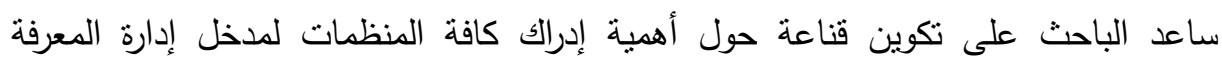

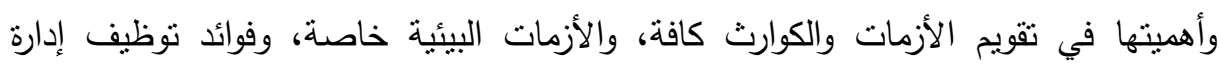
المعرفة لديها.

\section{همطط التوراسم}

بناءا على ما سبق فقد أرتأى الباحث أن دراسة موضوع هذا البحث تتطلب أن يقسم إلى هلى

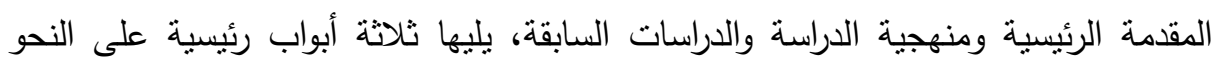
التالي :

الباب الأول: دور إدارة المعرفة في التقليل من آثار المخاطر البيئية الفصل الأول: أهمية إدارة المعرفة للصد من المخاطر البيئية

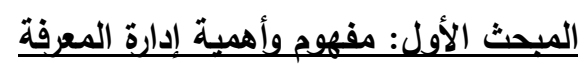

$$
\begin{aligned}
& \text { • المطلب الأول: تعريف المعرفة }
\end{aligned}
$$

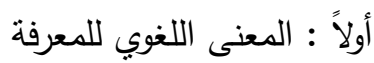

$$
\begin{aligned}
& \text { ثانياً : المعنى الإصطلاحي للمعرفة } \\
& \text { ثالثاً: العلاقة بين المعرفة والمعلوماتية } \\
& \text { المطلب الثاني : إدارة المعرفة وأهميتها } \\
& \text { أولاً: المقصود بإدارة المعرفة } \\
& \text { ثانياً: أهية إدارة المعرفة }
\end{aligned}
$$


المبحث الثاني: دور إدارة المعففة في الارتقاء بمستوى الأداء • المطلب الأول : نقل المعرفة ودورها في الارتقاء بمستوى الأداء

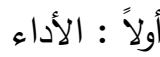

ثانياً : نقل المعرفة • المطلب الثاني: آليات الاستفادة من إدارة المعرفة في الارتقاء بالعاملين أولاً : أبعاد إدارة المعرفة وعلاقتها بالأداء ثانيًا : وظائف إدارة المعرفة ثالثًا : العوامل المؤثرة في تطور إدارة المعرفة

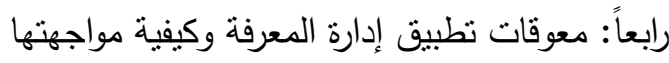

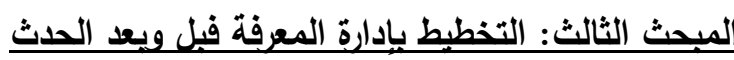
• المطلب الأول : نتظيم ومنهجية عمل مراكز القيادة الميدانية

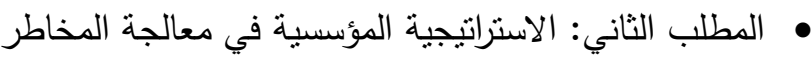
الفصل الثاني: المخاطر البيئية الناتجة عن الحريق المبحث الأول: نظرية الاشتعال وآليته • المطلب الأول: عوامل الاحتراق • المطلب الثاني: كيفية انتقال الحرارة بين الأجسام الأنراف المبحث الثاني: أسبـاب الحرائق وأنواعهيا • المطلب الأول: أسباب الحريق • المطلب الثاني: تصنيف وتقسيم الحرائق المبحث الثالث: الأخطار الناتجة عن الصرائق 
الباب الثاني: كيفية إدارة الأزمات والكوارث البيئية

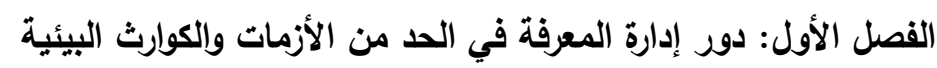

المبحث الأول: ماهية إدارة الأزمات وعلاقتها بالإدارة البيئية الإني • المطلب الأول: مفهوم الأزمة: الخصائص لـ . الأسباب .. الأنواع ع. .. المراحل

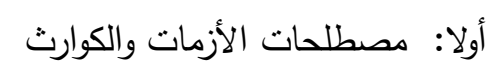

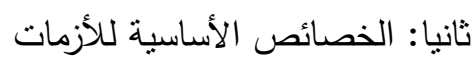

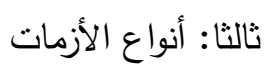
رابعا: أسباب نشوء الأزمات الأن الزمات خامساً: مراحل الأزمات الأرمات المبات • المطلب الثاني: إدارة الأزمات والكوارث وعلاقتتها بالإدارة البيئية أولا: مفهوم إدارة الأزمات وأهم أهدافها ثانياً: كيفية إعداد فريق لإدارة الأزمات الأنات ثالثاً: علاقة إدارة البيئة بإدارة الأزمات الأنات

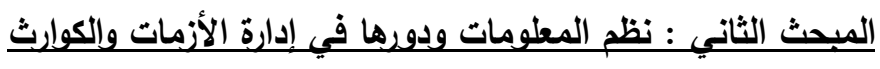

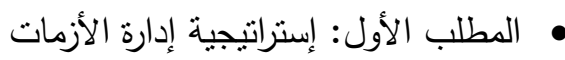

أولاً: الإستراتيجيات التقليدية الإنية ثانيا: الإستراتيجيات الحديثة ثالثا: عناصر استراتيجية إدارة الأزمات

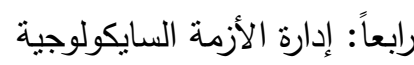
خامساً: إدارة الأزمة الإعلامية الإنمانية سادساً: التتسيق بين الأجهزة المعاونة في مواجهة الأزمات والكوارثة الإنهاث البيئية

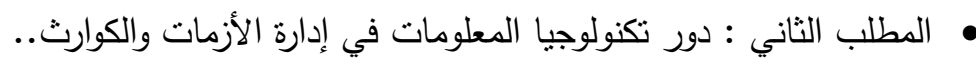

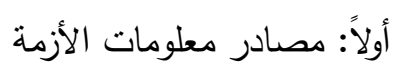
ثاناًاً: أنواع مصادر معلومات الأزمة الأنة ثالثاً: الثروط التي يجب مراعاعر مطاتها عند جمع معلومات الأزمة

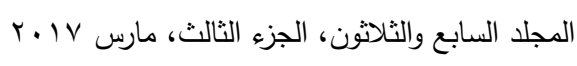


رابعاً: مدي الحاجة لتكنولوجيا المعلومات في العمل الأمني لإدارة الأزمة: خامساً: أمن نظام المعلومات لإدارة الأزمة سادساً: نظم المعلومات ودورها في صنع القرار المبحث الثالث : دور نظم الإنذار المبكر في دعم عملية التتبؤ بالأزمات

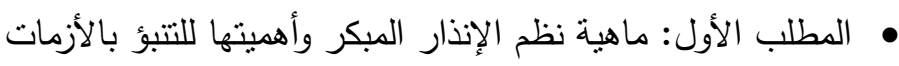
• المطلب الثاني: تقييم ووسائل قدرة المنظمة في مجال اكتثاف إنشارات الإنذار المبكر للأزمات أولا: مصادر معلومات نظم الإنذار المبكر ثانيا: تقييم قدرة المنظمة لإشارات الإنذار المبكر

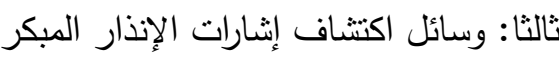

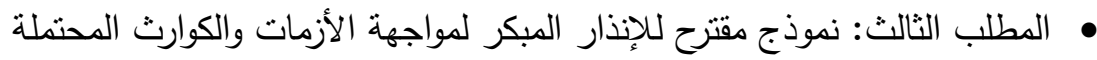

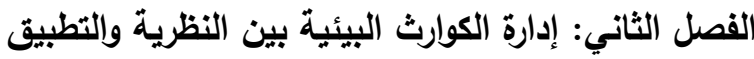

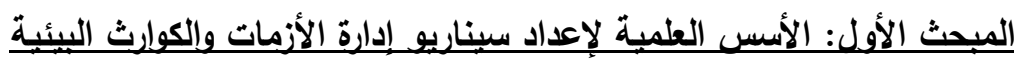

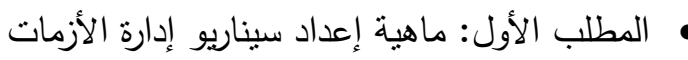

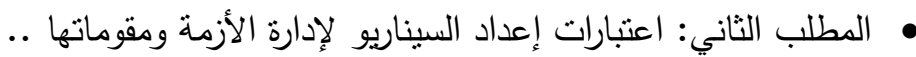

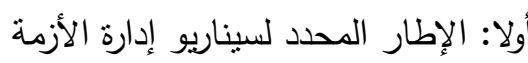
ثانيا: مقومات إعداد السيناريو

المبحث الثاني : معوقات التخطبط لمواجهة الأزمات والكوارث البيئية • المطلب الأول: تقريراً لمخاطر تحديد مصادر الكارثة المحتملة • المطلب الثاني: تقييم إجراءات الاستجابة الموجودة في حالة الأزمات والكوارث لُماثل

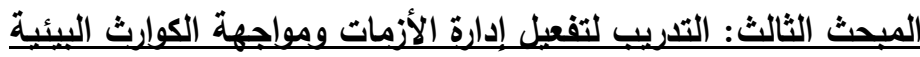
• المطلب الأول: مقترح إنشاء هيكل تتظيمي لإدارة الأزمات والكوارث • المطلب الثاني: تفعيل الوقاية والحماية .. إدارة مخاطر الكوارث البيئية 
الباب الثالث: حول تقويم إدارة الأزمات البيئية باستخدام إدارة المعرفة (الدراسة

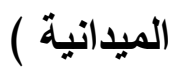

الفصل الأول: التعريف بمنهجية الاراسة

أولا: مقدمة الفمل

ثانيا: إعداد وتصميم قائمة الاسنقصاء

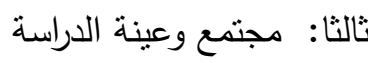

رابعا: توصيف متغيرات الدراسة : الاستة

خامسا: الأساليب الإحصائية لتحليل بيانات الدراسة

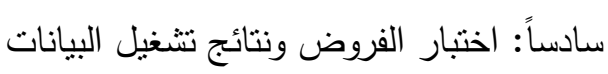

الفصل الثاني: استتتاجات ومستخلصات الاراسة

الخاتمة : وتحتوي على الاقتراحات والتوصيات الفيات

قائمة المراجع: وتحتوى على المراجع العربية والاجنيية

\section{أهمر نتمائج الصواسمة}

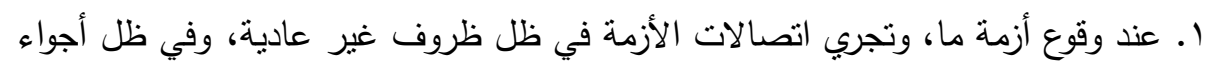

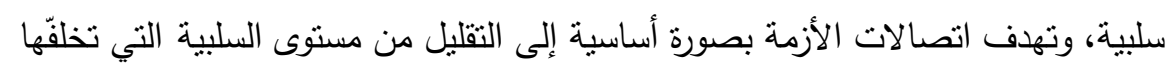

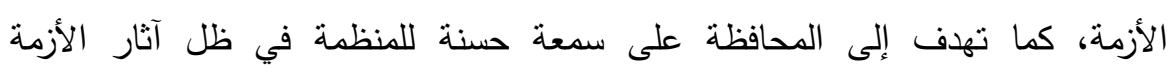

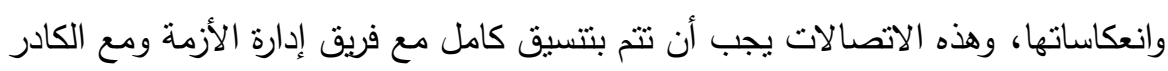

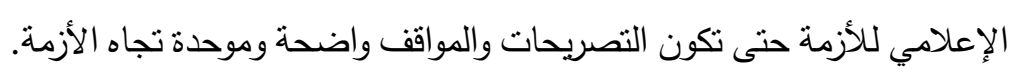

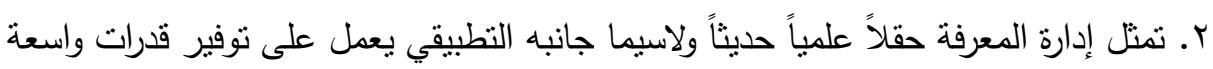

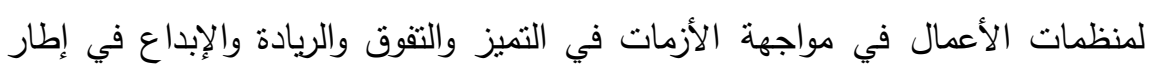

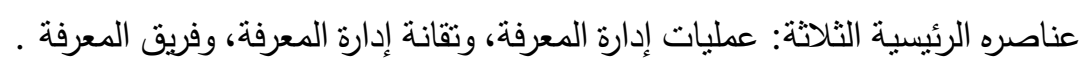

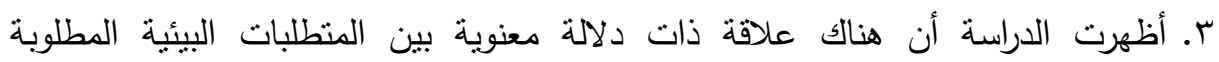
وإمكانيات المنشآت المادية والبشرية للحد من الحرائق 
ع. بينت الدراسة العملية وجود علاقة ارتباط قوية وذات دلالة معنوية للنظم المطبقة حاليا

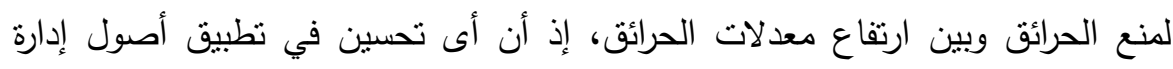

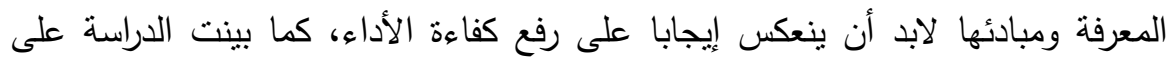
وجود علاقة بين كل عنصر من عناصر إدارة المعرفة وبين كل محور من محاور أداء

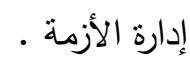

ه. وأوضحت الدراسة أنه قد يتم بالفعل تثكيل فرق عمل لإدارة الأزمات المختلفة، إلا أن عملية

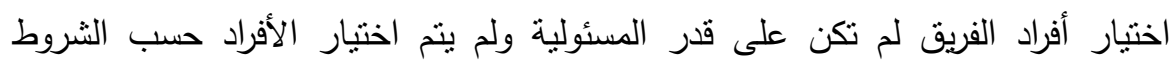
الموضوعة من قبل الإدارة العليا بشكل مسبق، مما يؤدي إلى فثل أفراد الفريق في تأدية المهام المنوطة بهم وإدارة الأزمة التي تواجههم بكفاءة وفعالية، وقد ترجع أسباب الفثل أيضًا إلى عدم وضوح خطط العمل، أو عدم وجود قنوات اتصال بين فريق العمل والإدارة العليا

للمنظمة.

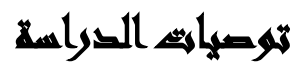

ا. إعداد دراسات أخرى مماتلة في كافة مجالات حوادث الحريق منل حوادث الحريق في

$$
\text { المصانع، حوادث الحريق في المزارع، وغيرها من المجالات. }
$$

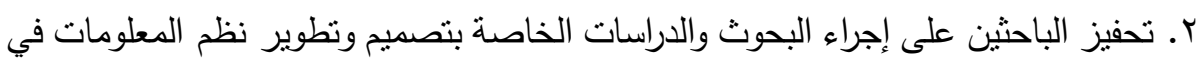
مجال حوادث الحريق في المنظمات.

r. إعطاء أهمية أكبر من قبل الإدارات العليا في منظمات الأعمال لإدارة المعرفة لتطوير

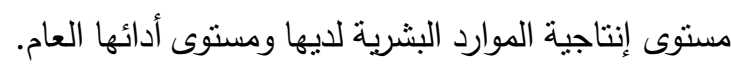
ع. استقطاب وتعيين مديري معرفة مؤهلين وقادرين على أداء أدوارهم المعرفية. ه. مراقبة كل ما هو مستجد في مجال تكنولوجيا المعلومات واستخدام نظم المعلومات الإدارية

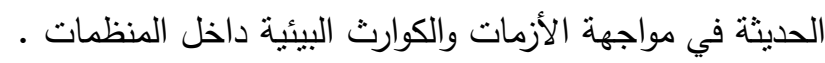

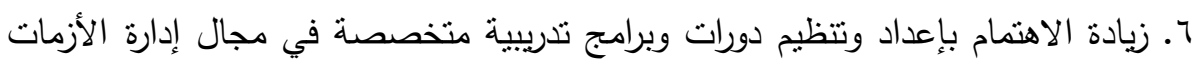
للمديرين والعاملين، تتصف بالاستمرارية من أجل تتمية وتأصيل المهارات والقدرات الإدارية بادية

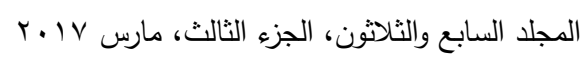


والسلوكية لإدارة الأزمات. - (المات.

V. الاستفادة من تجارب وخطط وممارسات التعامل مع الأزمات والكوارث البيئية في الدول

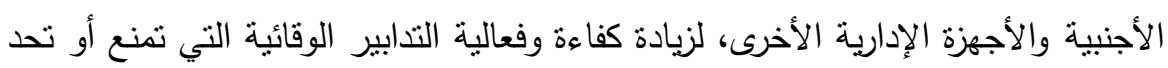
من الاحتمالات المستقبلية لحدوث الأزمة، العمل على وجود برامج محددة للتقبييم والتحليل

$$
\text { المستمر للأزمات والمخاطر المحتملة. }
$$

^. ضرورة إنشاء وحدة إدارية مستقلة لإدارة الأزمات في كل منظمة، تتبع الإدارة العليا في

$$
\text { قراراتها، وتكون مسؤولة بشكل مباشر عن علاج الأزمات المحتملة . }
$$

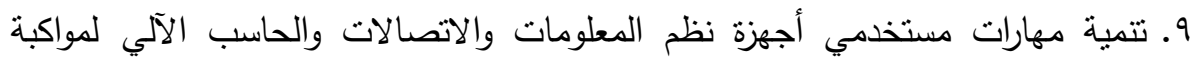
النطورات الحديثة في المجالات المختلفة ذات العلاقة بإدارة الأزمات البيئية في المنظمات.

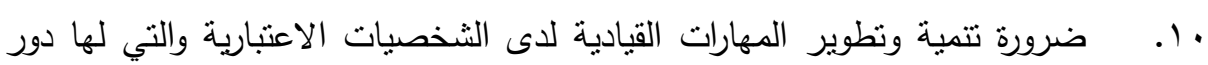
كبير وفعال في التعامل مع الأزمات وإداراتها .

\section{المراجـ}

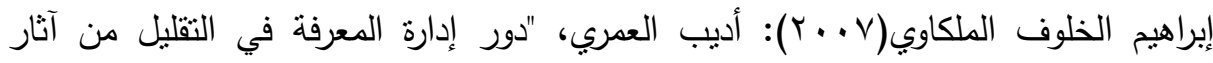

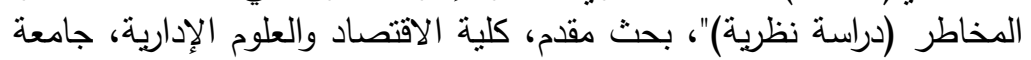

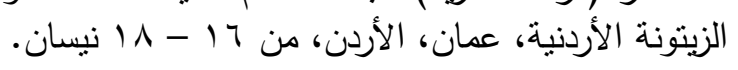

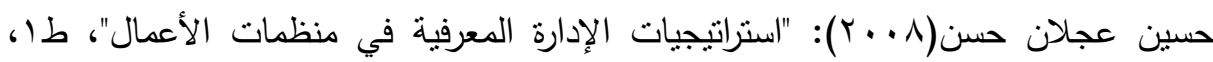
إثراء للنشر والتوزيع، الأردن، عمان.

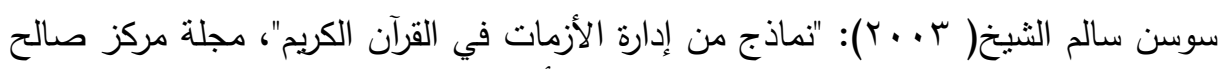
كامل للاقتصاد الإسلامي، جامعة الأزهر ، القاهرة.

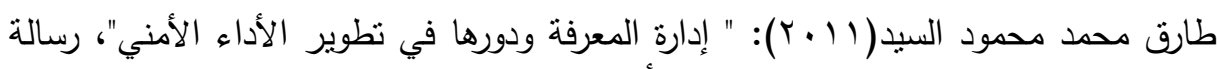
دكتوراه، قسم علوم الثرطة، أكاديمية الثرطة، كلية الدرأية الدراسات العليا، القاهرة.

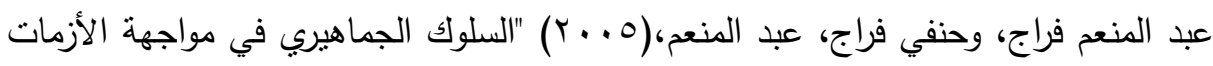

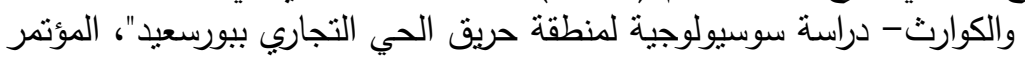

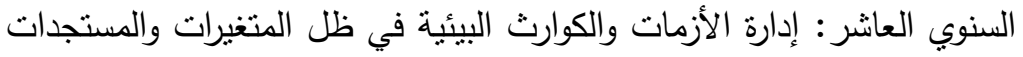

العالمية المعاصرة، المجلد الأول، القاهرة: دار الضيافة الضئة -جامعة عين شمس.

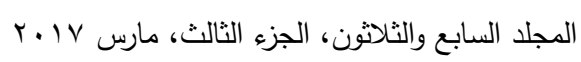


وسيم ميخائيل(0 . ـ ؟): "دور الوعي الاجتماعي في مواجهة الأزمات والكوارث البيئية - دراسة

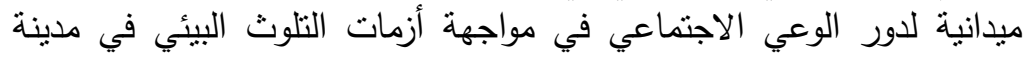

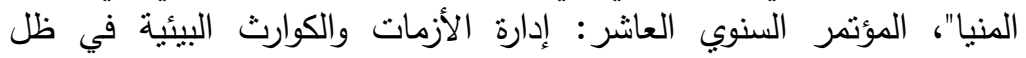

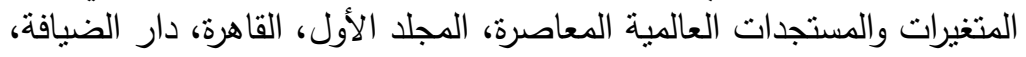

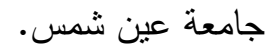

محمد عواد الزيات(^ . . ץ): " اتجاهات معاصرة في إدارة المعرفة"، طا، دار صفاء للنشر

والتوزيع، عمان.

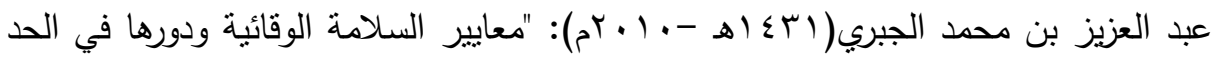

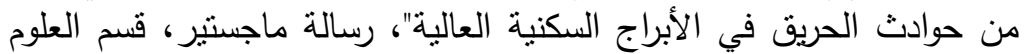

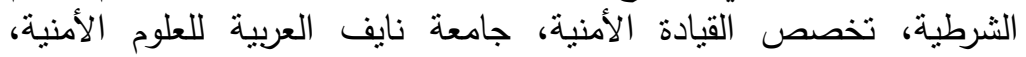

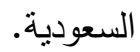

AMERICAN LIBRARY ASSOCIATION (ALA). Disaster response: a selected annotated bibliography (ALA Library Fact Sheet) compiled June 2001 is available at:

http://www.ala.org/ala/alalibrary/libraryfactsheet/alalibraryfactsheet10. htm.

Carolyn, B, "Validating Aunified Framework for knowledge management",

2002.WWW./CASit.org/km/kmrt/may02/index.htm.

Brent,w, "Chaos, crises and disasters: A strategic approach to crisis management in the tourism industry", tourism management, 25, 2004.

Clifford Geertz, "Knowledge management for technological innovation in organizations: The fusion process for creating intellectual capital", American Philosophical Association Institute of Philosophy, London, Common Knowledge 12 (3), 2006.

DORGE, V. and JONES, S.L, "Building an emergency plan: a guide for museums and other cultural institutions", Los Angeles, CA: Getty Conservation Institute, 1999.

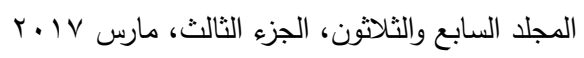


IRACI, Jo. Disaster recovery of modern information carriers: compact discs, magnetic tapes and magnetic disks. Ottawa: Canadian Conservation Institute (CCI), 2002. (Technical bulletin, 25). $16 \mathrm{p}$.

Lioret Francisco \& Delgado, " Statistical analysis of fire frequency models for Catalonia", International Journal of Wild land fire 13(1), 2005, Pp98 - 99.

WELLHEISER, Johanna and SCOTT, Jude, "An ounce of prevention: integrated disaster planning for archives, libraries and record centers", 2nd ed. Lanham, MD, Scarecrow Press/Ottawa: Canadian Archives Foundation, XVI, 2002.

\title{
EVALUATING ENVIRONMENTAL CRISES USING KNOWLEDGE MANAGEMENT A CASE STUDY ON CIVIL PROTECTION
}

Khalifa, M. A. ${ }^{(1)}$; Nada, M. A. ${ }^{(2)}$ and Abd ElHafz, A. R. ${ }^{(3)}$ 1) Faculty of Commerce, Ain Shams University. 2) American University 3) Institute of Environmental Studies and Research, Ain Shams University

\begin{abstract}
The crises looming often on the horizon of the organization, and be able to successful organizations through effective planning using knowledge management to avoid most of the crisis, but some of the events and unforeseen circumstances which are not planned by the organization effectively predicted great damage even the largest organizations, where it is crisis management, including lead one of the very important areas of services and associated with people's lives and property, and that you need to protect and provide continuous and rapid help .With the rapid expansion of cities and increasing population numbers, as well as various commercial and economic activities of

$$
\text { المجلد السابع والثلاثون، الجزء الثالث، مارس Y. r. r }
$$


crisis management has been dealing with many of the incidents and that different ways of combating the incident to another depending on the location and the causes of accidents and the material contained in the facility respecially the crises that occur due to the outbreak of fires.

If this study is to clarify the importance of information systems in the face of disasters and environmental crises, especially those relating to incidents of fires in the organizations, the Note and explore the events and circumstances unforeseen is a fundamental duty of the duties of directors, and management can expect crises and disasters that you may encounter private crises and environmental disasters, and considering how to deal with it by putting the worst case scenario, and develop attitudinal plans, and may be organized more sophisticated if they have the tools, methods and information systems that enable them to carry out planning to cope with crises and environmental disasters, and to give a comprehensive picture of how crises and disasters and environmental management using knowledge management has Taatdment comprehensive study to clarify the role of knowledge management.

The study included the concept and importance of knowledge management and its role in raising the level of performance, and indicate the role of knowledge management in minimizing the effects of the risks before and after the event, with a clarification of the environmental hazards caused by the fires and their causes and types of the outbreak, as the study active role for crisis management in the face of crises and disasters explained environmental 'where the study focused on the definition of the concept of crisis and its causes, types and stages, and the definition of concepts related and overlapping with it, as the study focused on identifying the nature of crisis management, as well as the statement of the role of information systems in crisis and environmental disaster management and explain the importance of effective training using knowledge management to activate the role played by the crisis management in the face of environmental disasters and crises within the organization.

$$
\text { المجلد السابع والثلاثون، الجزء الثالث، مارس VI ا. r }
$$


The study concluded several results of the most important and effective role played by environmental crises in the Organization's management as it one of the interactive systems to prevent or cope with fire incidents, as well as the proper planning of the use of information systems instrumental in crises and disasters and environmental management and crisis and environmental disaster management scenario on the basis of sound science. 